\title{
Extended Search for the Invisible Axion with the Axion Dark Matter Experiment
}

T. Braine, ${ }^{1}$ R. Cervantes, ${ }^{1}$ N. Crisosto, ${ }^{1}$ N. Du $\odot,{ }^{1,}$ S. Kimes, ${ }^{1}$ L. J. Rosenberg, ${ }^{1}$ G. Rybka, ${ }^{1}$ J. Yang,,${ }^{1}$ D. Bowring, ${ }^{2}$ A. S. Chou, ${ }^{2}$ R. Khatiwada, ${ }^{2}$ A. Sonnenschein, ${ }^{2}$ W. Wester, ${ }^{2}$ G. Carosi, ${ }^{3}$ N. Woollett, ${ }^{3}$ L. D. Duffy, ${ }^{4}$ R. Bradley, ${ }^{5}$ C. Boutan, ${ }^{6}$ M. Jones, ${ }^{6}$ B. H. LaRoque, ${ }^{6}$ N. S. Oblath, ${ }^{6}$ M. S. Taubman, ${ }^{6}$ J. Clarke, ${ }^{7}$ A. Dove, ${ }^{7}$ A. Eddins, ${ }^{7}$ S. R. O'Kelley, ${ }^{7}$ S. Nawaz, ${ }^{7}$ I. Siddiqi, ${ }^{7}$ N. Stevenson, ${ }^{7}$ A. Agrawal, ${ }^{8}$ A. V. Dixit, ${ }^{8}$ J. R. Gleason, ${ }^{9}$ S. Jois, ${ }^{9}$ P. Sikivie, ${ }^{9}$ J. A. Solomon, ${ }^{9}$ N. S. Sullivan, ${ }^{9}$ D. B. Tanner, ${ }^{9}$ E. Lentz, ${ }^{10}$ E. J. Daw, ${ }^{11}$ J. H. Buckley, ${ }^{12}$ P. M. Harrington, ${ }^{12}$ E. A. Henriksen, ${ }^{12}$ and K. W. Murch ${ }^{12}$

\section{(ADMX Collaboration)}

\author{
${ }^{1}$ University of Washington, Seattle, Washington 98195, USA \\ ${ }^{2}$ Fermi National Accelerator Laboratory, Batavia, Illinois 60510, USA \\ ${ }^{3}$ Lawrence Livermore National Laboratory, Livermore, California 94550, USA \\ ${ }^{4}$ Los Alamos National Laboratory, Los Alamos, California 87545, USA \\ ${ }^{5}$ National Radio Astronomy Observatory, Charlottesville, Virginia 22903, USA \\ ${ }^{6}$ Pacific Northwest National Laboratory, Richland, Washington 99354, USA \\ ${ }^{7}$ University of California, Berkeley, California 94720, USA \\ ${ }^{8}$ University of Chicago, Chicago, Illinois 60637, USA \\ ${ }^{9}$ University of Florida, Gainesville, Florida 32611, USA \\ ${ }^{10}$ University of Göttingen, Göttingen 37077, Germany \\ ${ }^{11}$ University of Sheffield, Sheffield S3 7RH, United Kingdom \\ ${ }^{12}$ Washington University, St. Louis, Missouri 63130, USA
}

(Received 30 October 2019; revised manuscript received 23 January 2020; accepted 18 February 2020; published 11 March 2020)

\begin{abstract}
This Letter reports on a cavity haloscope search for dark matter axions in the Galactic halo in the mass range 2.81-3.31 $\mu \mathrm{eV}$. This search utilizes the combination of a low-noise Josephson parametric amplifier and a large-cavity haloscope to achieve unprecedented sensitivity across this mass range. This search excludes the full range of axion-photon coupling values predicted in benchmark models of the invisible axion that solve the strong $C P$ problem of quantum chromodynamics.
\end{abstract}

DOI: 10.1103/PhysRevLett.124.101303

Axions are a hypothesized particle that emerged as a result of the Peccei-Quinn solution to the strong $C P$ problem [1-3]. In addition, axions are a leading dark matter candidate that could explain $100 \%$ of the dark matter in the Universe [4-8]. There are a number of mechanisms for the production of dark matter axions in the early Universe $[5,6,9,10]$. For the case where $\mathrm{U}_{\mathrm{PQ}}(1)$ becomes spontaneously broken after inflation, cosmological constraints suggest an axion mass on the scale of $1 \mu \mathrm{eV}$ or greater [11-16]. Two benchmark models for the axion are the Kim-ShifmanVainshtein-Zakharov (KSVZ) [17,18] and Dine-FischlerSrednicki-Zhitnitsky (DFSZ) $[19,20]$ models. Of the two, the DFSZ model is especially compelling because of its grand unification properties [19].

Published by the American Physical Society under the terms of the Creative Commons Attribution 4.0 International license. Further distribution of this work must maintain attribution to the author(s) and the published article's title, journal citation, and DOI. Funded by SCOAP .
The Axion Dark Matter eXperiment (ADMX) searches for dark-matter axions using an axion haloscope [21], which consists of a microwave-resonant cavity inside a magnetic field. In the presence of an external magnetic field, axions inside the cavity can convert to photons with frequency $f=E / h$, where $E$ is the total energy of the axion, including the axion rest-mass energy, plus a small kinetic energy contribution. The power expected from the conversion of an axion into microwave photons in the ADMX experiment is extremely low, $\mathcal{O}\left(10^{-23} \mathrm{~W}\right)$, requiring the use of a dilution refrigerator and an ultralow-noise microwave receiver to detect the photons.

In limits set in a previous Letter, ADMX became the only axion haloscope to achieve sensitivity to both benchmark axion models for axion masses between 2.66 and $2.81 \mu \mathrm{eV}$ [22]. This Letter reports on recent operations which extend the search for axions at DFSZ sensitivity to $2.66-3.31 \mu \mathrm{eV}$.

The ADMX experiment consists of a $136 \mathrm{~L}$ cylindrical copper-plated cavity placed in a 7.6 T field produced by a superconducting solenoid magnet. A magnetic field-free 
region above the cavity is maintained by a counterwound bucking magnet above the cavity. Field-sensitive receiver components, such as a Josephson parametric amplifier (JPA) and circulators, are located there, and the JPA is protected by additional passive magnetic shielding [23,24]. The resonant frequency of the cavity is set by two copper tuning rods that run parallel to the axis of the cavity and can be positioned near the center of the cavity or the walls of the cavity. Cryogenic gearboxes connect the tuning rods to room-temperature stepper motors, which tune the cavity during operations. When the frequency of the cavity is tuned to the same frequency as the photon produced from the axion, the expected power deposited into the cavity is [25]

$$
P_{\text {axion }}=2.2 \times 10^{-23} \mathrm{~W}\left(\frac{V}{136 \mathrm{~L}}\right)\left(\frac{B}{7.6 \mathrm{~T}}\right)^{2}\left(\frac{C}{0.4}\right) \times\left(\frac{g_{\gamma}}{0.36}\right)^{2}\left(\frac{\rho_{a}}{0.45 \mathrm{GeV} \mathrm{cm}^{-3}}\right)\left(\frac{f}{740 \mathrm{MHz}}\right)\left(\frac{Q}{30000}\right) .
$$

Here $V$ is the volume of the cavity; $B$ is the magnitude of the external magnetic field; $g_{\gamma}$ is the model-dependent axion-photon coupling, which has a value of $-0.97(0.36)$ for KSVZ (DFSZ) axions; $\rho_{a}$ is the local dark matter density; $f$ is the frequency of the photon; $Q$ is the loaded quality factor of the cavity; and $C$ is the form factor of the cavity.

The form factor reflects the overlap between the electric field of the cavity mode and the external magnetic field generated by the solenoid [25]. In the case of the ADMX cavity, the optimal form factor is observed with the $\mathrm{TM}_{010^{-}}$ like lowest-order tunable mode. Over the mass range explored in this Letter, the average form factor is 0.4. Several mode crossings between the $\mathrm{TM}_{010}$ mode frequency and weakly tuning TE or TEM modes occurred during operations, causing a significant drop in the form factor due to mode mixing. These were filled in using alternative rod configurations which reduced the form factor by only a moderate amount.

The signal-to-noise ratio for power within the experiment is set by the Dicke radiometer equation [26]:

$$
\frac{S}{N}=\frac{P_{\text {axion }}}{k_{B} T_{\text {sys }}} \sqrt{\frac{t}{b}}
$$

where $T_{\text {sys }}$ is the system noise temperature, equal to the combined physical temperature of the cavity and the noise temperature of the receiver chain in the Rayleigh-Jeans limit of the blackbody distribution; $t$ is the integration time; and $b$ is the detection bandwidth.

An antenna inserted into the cavity extracts power from the cavity and transfers it into the cryogenic receiver chain shown in Fig. 1. The power is amplified by a JPA with a tunable resonance, as demonstrated in Ref. [27], followed by a cryogenic heterostructure field-effect transistor (HFET) amplifier. The JPA used by ADMX was developed at UC Berkeley [28]. The JPA was operated in phasepreserving mode by pumping with a microwave tone $375 \mathrm{kHz}$ detuned from the cavity resonance. The resonant frequency of the JPA was tuned with a DC bias current supplied to a superconducting coil coupled to the SQUIDs in the JPA [29]. During operations, the JPA achieved a power gain of at least $20 \mathrm{~dB}$ over an instantaneous bandwidth of $10-20 \mathrm{MHz}$ and was tunable over the full bandwidth explored by the experiment.

To reduce the noise temperature, the cavity and JPA are cooled with a Janis Research dilution refrigerator. The mixing chamber of the dilution refrigerator is mounted to the top of the cavity, and its high cooling power enables ongoing operation of the cavity and JPA at temperatures on the order of $100 \mathrm{mK}$. Ruthenium oxide temperature sensors measured the cavity temperature to be typically $130 \mathrm{mK}$, and the temperature of the receiver amplifier package was $230 \mathrm{mK}$. The higher temperature in the region of the JPA was due to weak thermal contact between the cryogenic

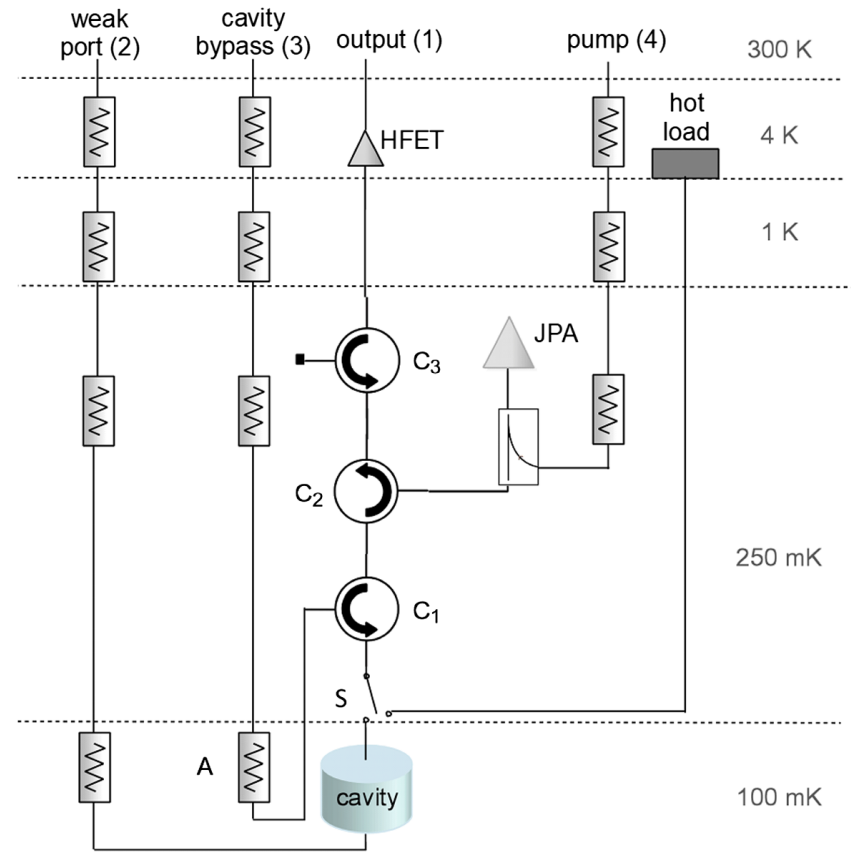

FIG. 1. The ADMX cryogenic receiver chain. $C_{1}, C_{2}$, and $C_{3}$ are circulators. Power is transmitted into the weak port (2) and cavity bypass lines (3) for transmission and reflection measurements from the cavity, respectively. The pump line (4) is used to supply a pump tone into the JPA. Switch $S$ can be toggled between the cavity and the hot load for noise calibration measurements. 
receiver package and the $4 \mathrm{~K}$ liquid helium reservoir surrounding the magnetic-field-free region.

Data were collected between January and October 2018. During standard data-taking operations, small steps in tuning rod position were taken to adjust the resonant frequency of the cavity. The cavity resonant frequency and $Q$ were measured with a $S_{12}$ measurement measuring the transmission through the cavity. A 50-kHz-wide power spectrum, centered on the cavity resonant frequency, was constructed by integrating the voltage time series from the cavity with a digitizer for 100 seconds. Periodically during data taking, the coupling of the antenna to the cavity mode was determined with an $S_{13}$ measurement to measure the power reflected from the cavity antenna. If the resonant absorption by the cavity was not at least $5 \mathrm{~dB}$, the antenna position was adjusted.

During data taking, synthetically generated axion signals were occasionally injected into the weakly coupled antenna. The frequencies of these synthetic axion injections were blinded to the group searching for axion candidates in the data.

The system noise temperature was measured in a twostep process. The noise contribution from the HFET amplifiers and warm receiver chain were calibrated from a $Y$-factor measurement, followed by a signal-to-noise improvement measurement to determine the noise contribution from the JPA [30]. For the $Y$-factor measurement, the JPA was unpowered, where it operated as a passive mirror, and switch $S$ was toggled between the cavity and a dedicated hot load. As shown in Fig. 1, when toggled to the cavity, thermal photons from attenuator $A$ were transmitted through circulator $C_{1}$ and reflected off the cavity. Attenuator $A$ was thermally sunk to the cavity and could be varied between $100 \mathrm{mK}$ and $1 \mathrm{~K}$. When toggled to the dedicated load, the thermal background was dominated by the hot load, which was heated between 4 and $6 \mathrm{~K}$. The power from the hot load was attenuated by switch $S$ and circulators $C_{1}, C_{2}$, and $C_{3}$. These were kept at the same cryogenic stage as the JPA and could be varied between 200 and $500 \mathrm{mK}$, independent of the cavity and dedicated load. These measurements were repeated 4 times during the datataking period. The measurements were combined and fitted to a model of the RF system, which yielded a downstream (of all components beyond the JPA electronics space) noise temperature of $11.3 \pm 0.1 \mathrm{~K}$ over the band of interest which was within expectations for the HFET amplifier operating in a high magnetic field [31]. These measurements also identified the attenuation between the cavity and the JPA to be $1.52 \pm 0.02 \mathrm{~dB}$, which was incorporated into the calculation of axion sensitivity as an attenuation of the signal from the cavity. This attenuation was consistent with the expected loss in the circulators, cables, and JPA below $780 \mathrm{MHz}$, and was larger above $780 \mathrm{MHz}$, as expected from the circulator specifications.

The signal-to-noise ratio improvement (SNRI) measurement consisted of measuring the increase in the digitized power from the cavity and the gain of the receiver with and without the JPA pump tone active. The gain of the JPA multiplied by the ratio of the power spectral density measurements yielded the ratio of the total system noise temperature to the noise temperature of the HFET and downstream components, such that

$$
T_{\text {sys }}=\frac{G_{\text {off }}}{G_{\text {on }}} \frac{P_{\text {on }}}{P_{\text {off }}} T_{\text {hfet }}=\frac{T_{\text {hfet }}}{S N R I},
$$

where $G$ was the transfer function at the desired frequency, and $P$ was the power at the desired frequency. A typical SNRI was $15.5 \mathrm{~dB}$. Because the total system noise temperature with the HFET $(11.3 \mathrm{~K})$ and cavity $(0.13 \mathrm{~K})$ was $11.43 \mathrm{~K}$, this SNRI corresponded to a typical operating system noise temperature at the input of the JPA of $320 \mathrm{mK}$.

The system noise was monitored by SNRI measurements roughly every 10 minutes and at eight different nearby bias currents and pump-power combinations, which were then updated for the optimal JPA SNRI.

The initial axion search was performed with the tuning rods in a symmetric configuration, and in that configuration eight mode crossings were observed. These crossings were identified with simulations and confirmed with wide-span $S_{12}$ transmission measurements. During initial data taking, the frequency ranges near these mode crossings were skipped over because of their poor form factors.

To fill in mode crossings, an antisymmetric rod configuration was used. In this rod configuration, many weakly tuning modes were shifted by several $\mathrm{MHz}$, shifting the positions of the corresponding mode crossings. Examples of both rod configurations are shown in Fig. 2. At frequency ranges previously covered by mode crossings, the form factor dropped to about 0.35 , which was sufficient for axion searches. Three mode crossings were covered by this method. The five remaining mode crossings were either too wide or the interfering mode could not be shifted, so that we were unable to obtain sufficient sensitivity to the axion to set limits at those frequencies.

The analysis procedures followed those discussed in Ref. [32]. The goal of the analysis was to average the individual spectra into a single grand spectrum to increase the signal-to-noise ratio of possible axion signals, and then search for candidate axion signals. First, the receiver shape was removed with a fit to a six-order Padé approximation. The power was scaled to the system noise temperature and weighted by the cavity $Q$ to convert the spectrum into a measurement of the power in excess of the noise. This spectrum was then filtered by a convolution with one of two different axion signal shapes: a boosted MaxwellBoltzmann line shape, predicted from the standard halo model for axion dark matter, as described in Ref. [33], with a local density of $0.45 \mathrm{GeV} / \mathrm{cm}^{3}$, or a line shape derived from $N$-body simulations described in Ref. [34], with a local density of $0.63 \mathrm{GeV} / \mathrm{cm}^{3}$. 


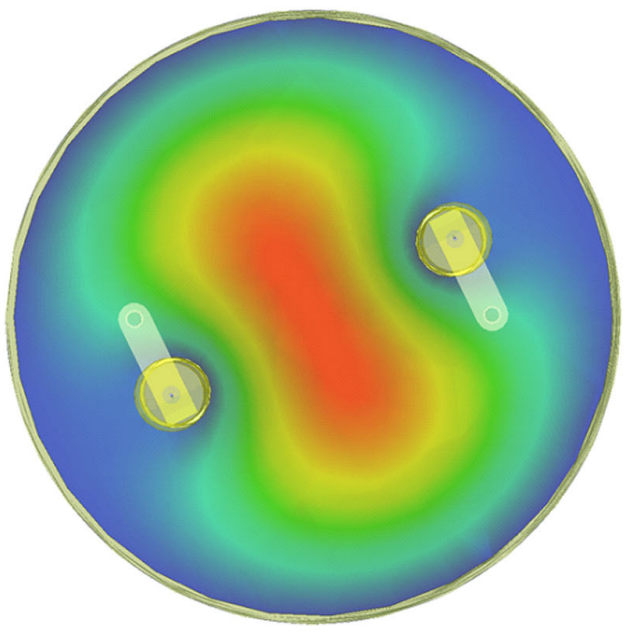

Symmetric

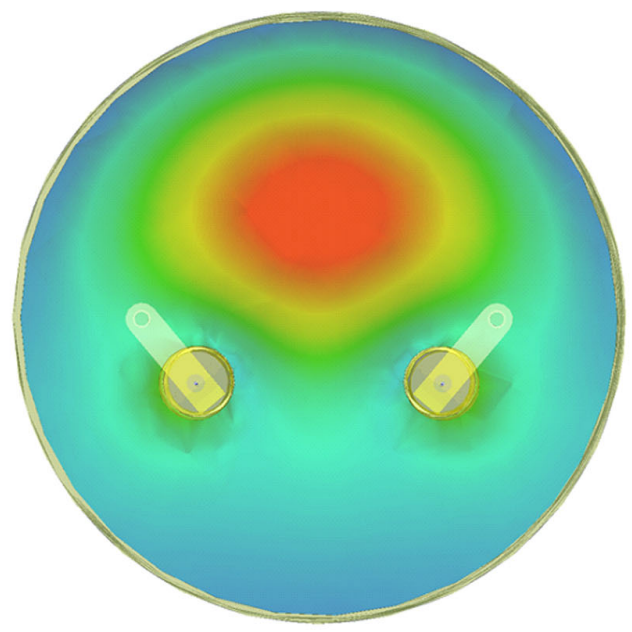

Anti-Symmetric

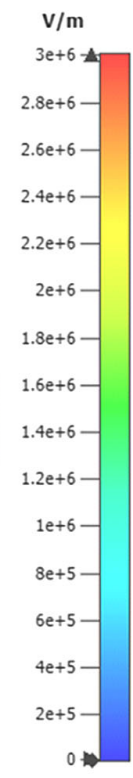

FIG. 2. A top-down view of the ADMX cavity. The resonant frequency of the cavity is set by the position of two tuning rods. During initial data taking, the rods are moved in a symmetric configuration (left). To scan over mode crossings, an antisymmetric configuration is used (right). The frequency of the $\mathrm{TM}_{010}$ mode is the same in both configurations shown. The colors indicate the magnitude of the electric field component along the axis of the cavity.

After a frequency range had been scanned with a more than sufficient signal-to-noise ratio to exclude DFSZ axions in the null case, "candidate" axion signals were identified. Frequencies with upward fluctuations in power exceeding $3 \sigma$, or that could not exclude the DFSZ axion coupling strength, were deemed "candidate" axion signals, requiring rescanning and further analysis. At frequencies with no statistically significant power excess, upper limits were placed on the axion-photon coupling using the measured power and uncertainty at that frequency.

Following the initial search, candidate frequencies were rescanned with significantly longer integration time to improve the expected signal-to-noise ratio for a possible axion. If the power at the candidate frequency did not persist and a DFSZ axion signal could be excluded, the candidate was determined to be transient. Frequencies that persisted past the second rescan were subjected to additional individual candidate checks.

The first step of individual candidate analysis was to check whether candidates were external radio signals detected within the experiment by measuring whether the signal power was maximized when the cavity frequency was tuned to the signal frequency. Axion signals would develop within the resonant cavity, so that the power from an axion signal would increase as the cavity was tuned to match the signal frequency. On the other hand, external interference would be picked up by components further along in the receiver chain, so that the signal power would be independent of the cavity frequency.
To test the detection efficiency of the analysis procedure, 20000 software injected signals were injected with powers between those expected for DFSZ and KSVZ axions, and the injected powers were compared to the powers detected by the analysis. It was found that the detected power was suppressed by a factor of 0.82 due to signal removal from the receiver spectral fit. This effect is accounted for in our reported limits.

Over the course of the axion search, ADMX searched for axions from 680-790 MHz. Within this range, three persistent signals were observed, at 686.310, 730.195, and $780.255 \mathrm{MHz}$. The first and last signals (686.310 and $780.255 \mathrm{MHz}$ ) did not maximize on resonance, indicating they were due to external radio interference and therefore could be excluded as axions.

The signal at $730.195 \mathrm{MHz}$ (Fig. 3) maximized on resonance and was consistent in power and linewidth to the signal expected from a DFSZ axion. This result triggered a decision to ramp the magnetic field down to determine whether the power of the signal would scale as $B^{2}$, in a manner consistent with an axion signal. Before the procedure was initiated, the candidate was revealed to be a synthetic axion signal. Instead, the synthetic injection was disabled, and the region around the candidate was rescanned. No signal appeared at $730.195 \mathrm{MHz}$, and thus all candidate axion signals were excluded. We concluded either that the axion is not within the explored range, that the axion dark-matter density is a small fraction of the halo density, or that the axion-photon coupling constant is significantly below the prediction for DFSZ. 


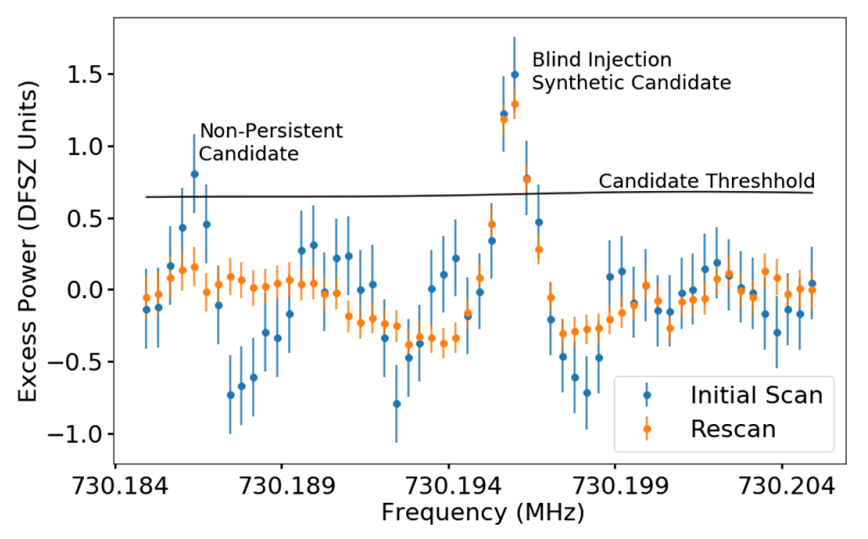

FIG. 3. An example of combined power spectra after a Maxwell-Boltzmann shape filter, with blue indicating the initial scan data and orange indicating data taken during a rescan with roughly 4 times more integration time. The prominent peak centered at $730.195 \mathrm{MHz}$ corresponds to a blind signal injection identified in the analysis that persisted after a rescan; the small peak to the left at $730.186 \mathrm{MHz}$ was a candidate that did not persist in the rescan. Because of a mismatch between the receiver spectral shape and the axion signal, the power at frequencies surrounding the candidate is suppressed by the receiver spectral background removal. This can be seen in the frequency background surrounding the $730.195 \mathrm{MHz}$ candidate in the rescan (orange) data.

Given the absence of axion-like signals, a 90\% upper confidence limit was set on the axion-photon coupling over the scanned mass range. Due to the loss of sensitivity at mode crossings, we do not report limits over some regions.
TABLE I. Primary sources of systematic uncertainty within the experiment. The total combined uncertainty was treated as an uncertainty on the total axion power from the cavity.

\begin{tabular}{lc}
\hline \hline Source & Fractional uncertainty \\
\hline $\mathrm{B}^{2} * \mathrm{~V}^{* \mathrm{f}}$ & 0.05 \\
$\mathrm{Q}$ & 0.011 \\
Coupling & 0.0055 \\
$\mathrm{RF}$ model fit & 0.029 \\
Temperature sensors & 0.05 \\
SNRI measurement & 0.042 \\
Total on axion power & 0.088 \\
\hline \hline
\end{tabular}

The fractional systematic uncertainties in the experiment are listed in Table I, which are modeled as uncertainties on the expected axion signal from the cavity. For models where axions make up $100 \%$ of dark matter, these limits exclude DFSZ axion-photon couplings between 2.66 and $3.31 \mu \mathrm{eV}$ for both isothermal sphere halo models and $N$-body simulations (Fig. 4). These results represent a factor-of- 4 increase in mass coverage over those reported in Ref. [22].

ADMX will utilize a similar cavity with larger tuning rods and improved thermalization between the dilution refrigerator and quantum amplifier package to continue to search dark-matter axions at higher masses with increased sensitivity. These future searches, built on current research and development [36,37], will probe even more deeply into the well-motivated yet unexplored axion parameter space.

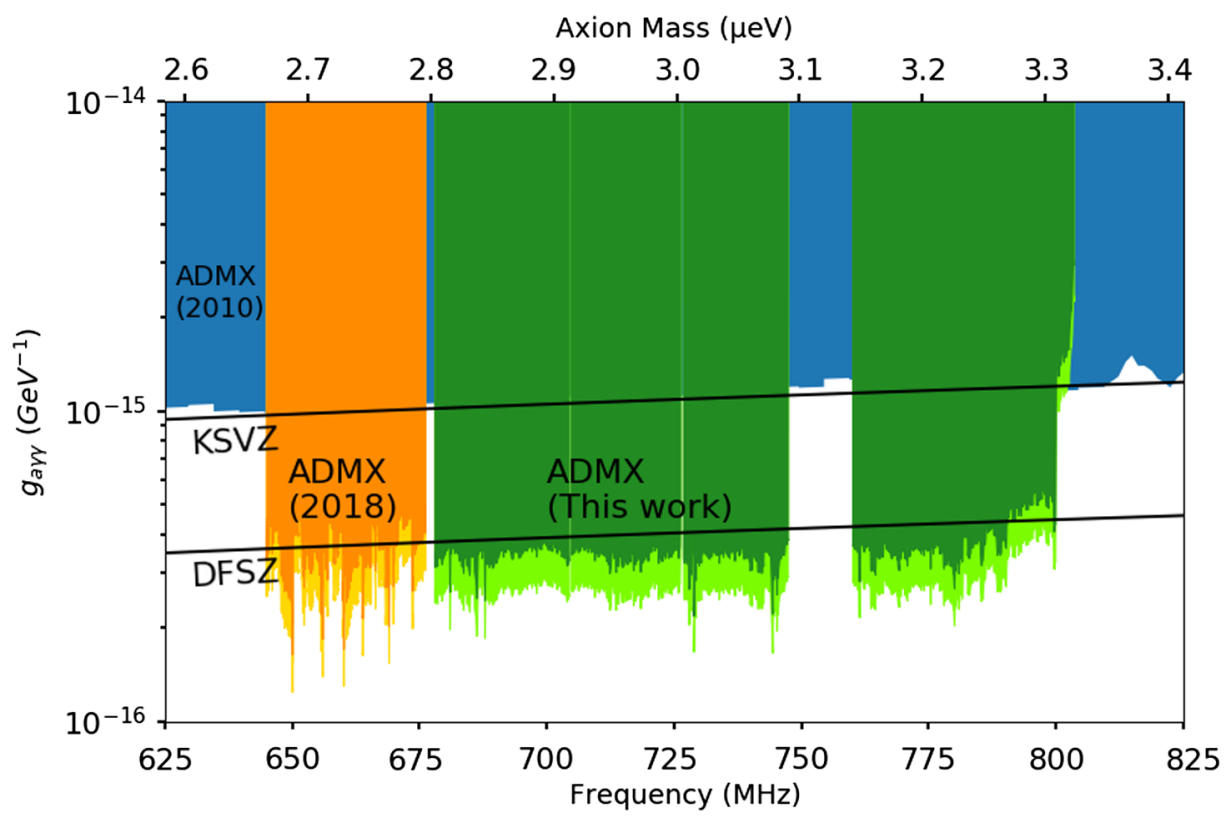

FIG. 4. 90\% confidence exclusion on axion-photon coupling as a function of axion mass for the Maxwell-Boltzmann (MB) darkmatter model and $N$-body model. Blue: Previous limits reported in Ref. [35]. Orange: Previous limits reported in Ref. [22]. Green: Limits from this work. Darker shades indicate limits set for the MB model [22,33], and lighter shades indicate limits set for the $N$-body model [22,34]. 
This work was supported by the U.S. Department of Energy through Grants No. DE-SC0009800, No. DESC0009723, No. DE-SC0010296, No. DE-SC0010280, No. DE-SC0011665, No. DEFG02-97ER41029, No. DEFG02-96ER40956, No. DEAC52-07NA27344, No. DEC03-76SF00098, and No. DE-SC0017987. Fermilab is a U.S. Department of Energy, Office of Science, HEP User Facility. Fermilab is managed by Fermi Research Alliance, LLC (FRA), acting under Contract No. DE-AC0207CH11359. Additional support was provided by the Heising-Simons Foundation and by the Lawrence Livermore National Laboratory and Pacific Northwest National Laboratory LDRD offices. LLNL Release No. LLNL-JRNL-806360.

*Corresponding author. ndu@uw.edu

[1] R. D. Peccei and H. R. Quinn, Phys. Rev. Lett. 38, 1440 (1977).

[2] S. Weinberg, Phys. Rev. Lett. 40, 223 (1978).

[3] F. Wilczek, Phys. Rev. Lett. 40, 279 (1978).

[4] P. A. R. Ade et al. (Planck Collaboration), Astron. Astrophys. 571, A16 (2014)

[5] L. Abbott and P. Sikivie, Phys. Lett. 120B, 133 (1983).

[6] M. Dine and W. Fischler, Phys. Lett. 120B, 137 (1983).

[7] J. Preskill, M. B. Wise, and F. Wilczek, Phys. Lett. 120B, 127 (1983).

[8] J. Ipser and P. Sikivie, Phys. Rev. Lett. 50, 925 (1983).

[9] P. Sikivie, Lect. Notes Phys. 741, 19 (2008).

[10] M. P. Hertzberg, M. Tegmark, and F. Wilczek, Phys. Rev. D 78, 083507 (2008).

[11] C. Bonati, M. D’Elia, M. Mariti, G. Martinelli, M. Mesiti, F. Negro, F. Sanfilippo, and G. Villadoro, J. High Energy Phys. 03 (2016) 155.

[12] E. Berkowitz, M. I. Buchoff, and E. Rinaldi, Phys. Rev. D 92, 034507 (2015).

[13] S. Borsanyi, Z. Fodor, J. Guenther, K.-H. Kampert, S. D. Katz, T. Kawanai, T. G. Kovacs, S. W. Mages, A. Pasztor, F. Pittler, J. Redondo, A. Ringwald, and K. K. Szabo, Nature (London) 539, 69 (2016).

[14] G. Ballesteros, J. Redondo, A. Ringwald, and C. Tamarit, Phys. Rev. Lett. 118, 071802 (2017).

[15] M. Dine, P. Draper, L. Stephenson-Haskins, and D. Xu, Phys. Rev. D 96, 095001 (2017).
[16] P. Petreczky, H.-P. Schadler, and S. Sharma, Phys. Lett. B 762, 498 (2016).

[17] J. E. Kim, Phys. Rev. Lett. 43, 103 (1979).

[18] M. A. Shifman, A. Vainshtein, and V. I. Zakharov, Nucl. Phys. B166, 493 (1980).

[19] M. Dine, W. Fischler, and M. Srednicki, Phys. Lett. 104B, 199 (1981).

[20] A. Zhitnitsky, Sov. J. Nucl. Phys. 31, 260 (1980).

[21] P. Sikivie, Phys. Rev. Lett. 51, 1415 (1983).

[22] N. Du et al. (ADMX Collaboration), Phys. Rev. Lett. 120, 151301 (2018).

[23] S. J. Asztalos, G. Carosi, C. Hagmann, D. Kinion, K. van Bibber, M. Hotz, L. J. Rosenberg, G. Rybka, J. Hoskins, J. Hwang, P. Sikivie, D. B. Tanner, R. Bradley, and J. Clarke, Phys. Rev. Lett. 104, 041301 (2010).

[24] S. Asztalos, G. Carosi, C. Hagmann, D. Kinion, K. van Bibber, M. Hotz, L. J. Rosenberg, G. Rybka, A. Wagner, J. Hoskins, C. Martin, N. S. Sullivan, D. B. Tanner, R. Bradley, and J. Clarke, Nucl. Instrum. Methods Phys. Res., Sect. A 656, 39 (2011).

[25] P. Sikivie, Phys. Rev. D 32, 2988 (1985).

[26] R. H. Dicke, Rev. Sci. Instrum. 17, 268 (1946).

[27] L. Zhong et al., Phys. Rev. D 97, 092001 (2018).

[28] I. Siddiqi, R. Vijay, F. Pierre, C. M. Wilson, M. Metcalfe, C. Rigetti, L. Frunzio, and M. H. Devoret, Phys. Rev. Lett. 93, 207002 (2004).

[29] J. Y. Mutus, T. C. White, E. Jeffrey, D. Sank, R. Barends, J. Bochmann, Y. Chen, Z. Chen, B. Chiaro, A. Dunsworth et al., Appl. Phys. Lett. 103, 122602 (2013).

[30] T. L. Wilson, arXiv:1111.1183.

[31] E. Daw and R.F. Bradley, J. Appl. Phys. 82, 1925 (1997).

[32] B. M. Brubaker, L. Zhong, S. K. Lamoreaux, K. W. Lehnert, and K. A. van Bibber, Phys. Rev. D 96, 123008 (2017).

[33] M. S. Turner, Phys. Rev. D 42, 3572 (1990).

[34] E. W. Lentz, T. R. Quinn, L. J. Rosenberg, and M. J. Tremmel, Astrophys. J. 845, 121 (2017).

[35] S. Asztalos, E. Daw, H. Peng, L. J. Rosenberg, C. Hagmann, D. Kinion, W. Stoeffl, K. van Bibber, P. Sikivie, N. S. Sullivan, D. B. Tanner, F. Nezrick, M. S. Turner, D. M. Moltz, J. Powell, M.-O. André, J. Clarke, M. Mück, and R. F. Bradley, Phys. Rev. D 64, 092003 (2001).

[36] C. Boutan, Ph.D. thesis, University of Washington, 2017.

[37] C. Boutan et al. (ADMX Collaboration), Phys. Rev. Lett. 121, 261302 (2018). 